\title{
BOUNDS FOR INDICES OF COINCIDENCE AND ENTROPIES
}

\author{
Ana Maria ACU* , Gülen BaşCAnbaZ-TunCA And IOAN RAŞA
}

\begin{abstract}
In this paper we consider a parameterized family of discrete probability distributions and investigate the Rényi,Tsallis, and Shannon entropies associated with them. Lower and upper bounds for these entropies are obtained, improving some results from the literature. The proofs are based on several methods from classical analysis, theory of dual cones, and the stochastic majorization theory. The Rényi and Tsallis entropies are naturally expressed in terms of the index of coincidence. Consequently we study in detail the index of coincidence associated to the corresponding discrete probability distributions. The obtained results lead immediately to properties of the entropies.
\end{abstract}

Mathematics subject classification (2010): 94A17, 26A51, 26D10, 39B62.

Keywords and phrases: Probability distribution, index of coincidence, bounds for entropies, convex functions, stochastic majorization technique.

\section{REFERENCES}

[1] J. A. Adell, A. Lekuona, Y. Yu, Sharp Bounds on the Entropy of the Poisson Law and Related Quantities, IEEE Transactions on Information Theory 56, 5 (2010), 2299-2306.

[2] K. Baumann, M. Heilmann, I. Rasa, Further Results for kth order Kantorovich Modification of Linking Baskakov type operators, Results. Math. 69 (2016), 297-315.

[3] A. BĂRAR, G. R. MocANU, I. RAŞA, Bounds for some entropies and special functions, Carpathian J. Math. 34, 1 (2018), 9-15.

[4] A. BĂRAR, G. MocAnU, I. RASA, Heun functions related to entropies, RACSAM 113 (2019), 819830.

[5] E. Berdysheva, Studying Baskakov-Durrmeyer operators and quasi-interpolants via special functions, J. Approx. Theory 149 (2007), 131-150.

[6] S. Chang AND E. Weldon, Coding for T-user multiple-access channels, IEEE Trans. Information Theory 25 (1979), 684-691.

[7] C. Elsner, M. Prévost, Expansion of Euler's constant in terms of Zeta numbers, J. Math. Anal. Appl. 398 (2013), 508-526.

[8] I. Gavrea, M. IVAn, On a conjecture concerning the sum of the squared Bernstein polynomials, Appl. Math. Comput. 241 (2014), 70-74.

[9] H. W. Gould, Combinatorical Identities - A standardized set of tables listing 500 binomial coefficient summations, Morgantown, 1972.

[10] S. Karlin, W. J. Studden, Tchebycheff Systems: With Applications in Analysis and Statistics, Interscience, New York, 1966.

[11] C. KNESSEL, Integral Representations and Asymptotic Expansions for Shannon and Renyi Entropies, Appl. Math. Lett. 11, 2 (1998), 69-74.

[12] J. OHLIN, On a class of measures of dispersion with application to optimal reinsurance, Astin Bull. 5, 2 (1969), 249-266.

[13] A. W. Marshall, I. Olkin, C. A. BARry, Inequalities: Theory of Majorization and Its Applications, Springer, New York, 2011.

[14] J. Mrowiec, T. RaJBA, S. WASOwicz, A solution to the problem of Raşa connected with Bernstein polynomials, J. Math. Anal. Appl. 446 (2017), 864-878. 
[15] T. RAJBA, On some recent applications of stochastic convex ordering theorems to some functional inequalities for convex functions: A Survey Developments in Functional Equations and Related Topics, (J. Brzdek, K. Cieplinski, and T. Rassias (Eds.)), Springer Optim. Appl. 124 (2017), 231-274.

[16] I. RAŞA, Entropies and Heun functions associated with positive linear operators, Applied Mathematics and Computation 268 (2015), 422-431.

[17] I. RAŞA, Complete monotonicity of some entropies, Period Math Hung 17 (2017), 159-166. 